\title{
Perfil dos recém-nascidos prematuros atendidos no seguimento ambulatorial em uma cidade do sul do Brasil
}

\author{
Profile of premature newborns cared in an outpatient follow-up in a city in southern Brazil \\ Perfil de recién nacidos prematuros atendidos en seguimiento ambulatorio en una ciudad \\ del sur de Brasil
}

Fernanda Fontana ${ }^{1}$, Igor Soares Vieira ${ }^{1}$, Luciano Dias de Mattos Souza ${ }^{1 *}$.

\begin{abstract}
RESUMO
Objetivo: Analisar o perfil de recém-nascidos prematuros atendidos em um seguimento ambulatorial em uma cidade do sul do Brasil. Métodos: Estudo observacional, retrospectivo, através de análise de prontuários. Foram incluídos todos os pré-termos com idade gestacional inferior a 35 semanas e após alta, tiveram acompanhamento regular até completar doze meses de idade corrigida, no período de janeiro de 2010 a dezembro de 2017. Resultados: Um total de 9419 prontuários foram revisados, entretanto apenas 397 preencheram os critérios de inclusão. Os prematuros acompanhados no ambulatório de pediatria tiveram um crescimento adequado e compatível com as curvas do Intergrowth-21. Na triagem neonatal, o teste da orelhinha apresentou maior alteração $(10,3 \%)$, seguido do teste do pezinho $(3,4 \%)$. As comorbidades mais prevalentes foram as alterações cardiológicas $(51,1 \%)$, alterações no desenvolvimento neuropsicomotor $(35,1 \%)$ e anemia $(30,5 \%)$. Prematuridade extrema, baixo peso ao nascer e apgar abaixo de 7 no quinto minuto foram os fatores de risco mais associados as comorbidades encontradas. Conclusão: Os resultados sugerem que os prematuros acompanhados no ambulatório de pediatria da universidade têm um crescimento compatível com as curvas do Intergrowth-21, entretanto, é imprescindível o acompanhamento a médio e longo prazo, especialmente nos casos de prematuridade extrema, baixo peso ao nascer e apgar baixo para diagnóstico e intervenção precoce das morbidades.
\end{abstract}

Palavras-chave: Recém-nascido prematuro, Idade gestacional, Fatores de risco.

\begin{abstract}
Objective: To analyze the profile of premature newborns treated in an outpatient follow-up in a city in southern Brazil. Methods: Observational, retrospective study, through analysis of medical records. All preterm infants with a gestational age of less than 35 weeks were included and, after discharge, had regular follow-up until completing twelve months of corrected age, from January 2010 to December 2017. Results: A total of 9419 medical records were reviewed, however only 397 met the inclusion criteria. Premature infants monitored at the pediatric outpatient clinic had adequate growth compatible with Intergrowth-21st curves. In neonatal screening, the ear test showed the greatest change (10.3\%), followed by the "teste do pezinho" $(3.4 \%)$. The most prevalent comorbidity was cardiac change (51.1\%). Extreme prematurity, low birth weight and having a score below 7 in the fifth minute were the risk factors most associated with the comorbidities found. Conclusion: The results suggest that preterm infants monitored at the university pediatric outpatient clinic have a growth compatible with the Intergrowth-21 curves, however, medium and long term follow-up is essential, especially in cases of extreme prematurity, low birth weight and apgar low for diagnosis and early intervention of morbidities.
\end{abstract}

Keywords: Infant, Premature, Gestational age, Risk factors.

\section{RESUMEN}

Objetivo: Analizar el perfil de prematuros atendidos en seguimiento ambulatorio en una ciudad del sur de Brasil. Métodos: Estudio observacional, retrospectivo, mediante análisis de historias clínicas. Se incluyeron todos los recién nacidos prematuros con una edad gestacional menor a 35 semanas y, luego del alta, se les realizó un seguimiento regular hasta completar los doce meses de edad corregida, desde enero de 2010 hasta

${ }^{1}$ Universidade Católica de Pelotas (UCPel), Pelotas - RS. `E-mail: luciano.dms@gmail.com 
diciembre de 2017. Resultados: Se revisaron un total de 9419 historias clínicas. sin embargo, solo 397 cumplieron los criterios de inclusión. Los bebés prematuros seguidos en la consulta externa de pediatría tuvieron un crecimiento adecuado y fueron compatibles con las curvas Intergrowth-21st. En el cribado neonatal, la prueba de oído mostró el mayor cambio (10,3\%), seguido de lo "teste do pezinho" (3,4\%). La comorbilidad más prevalente fue el cambio cardíaco $(51,1 \%)$. La prematuridad extrema, el bajo peso al nacer y tener un apgar por debajo de 7 en el quinto minuto fueron los factores de riesgo más asociados a las comorbilidades encontradas. Conclusión: Los resultados sugieren que los prematuros monitorizados en la consulta externa de pediatría de la universidad tienen un crecimiento compatible con las curvas Intergrowth21, sin embargo, el seguimiento a medio y largo plazo es fundamental, especialmente en casos de prematuridad extrema, bajo peso al nacer y apgar. baja para diagnóstico e intervención temprana de morbilidades.

Palabras clave: Lactante, Prematuro, Edad gestacional, Factores de riesgo.

\section{INTRODUÇÃO}

O nascimento prematuro é uma das principais causas de morbimortalidade neonatal em todo o mundo (LEHTONEN L, et al., 2017). Classicamente definido como o nascimento que ocorre antes da $37^{\text {a }}$ semana de gravidez, também é o principal responsável por deficiências adquiridas após o nascimento (WHO, 1977; TORCHIN H e ANCEL P, 2016). Exceto pelas malformações congênitas, 75\% das mortes perinatais e 50\% das anormalidades neurológicas são atribuídas diretamente aos prematuros (TORCHIN H e ANCEL P, 2016).

Graças aos avanços na tecnologia e melhorias nos cuidados de saúde, vários recém-nascidos prematuros sobrevivem com menos sequelas. No entanto, muitos deles permanecem vulneráveis a complicações de longo prazo que podem persistir por toda a vida (HEDDERICH DM, et al., 2020; HEDDERICH DM, et al., 2019).

Estudos revelam alguns dos desfechos associados aos recém-nascidos prematuros: distúrbios cognitivos, distúrbios de visão e audição, possíveis doenças crônicas e cardiopatias no futuro e até mesmo prováveis sequelas neurológicas (MAIA RRP e SOUZA JMP, 2010; MOREIRA NETO AR, et al., 2011).

Assim, o prematuro precisa de um número maior de avaliações do estado de saúde, e a alta hospitalar não significa a resolução dos problemas dessa população, tornando-se imperativo o seguimento ambulatorial especializado, com vista à detecção e intervenção precoces das intercorrências com atenção especial no acompanhamento do crescimento, nutrição, funções visuais, auditivas, neuromotoras, imunização e cuidado domiciliar (SAFARI S e HAMRAH MP, 2017; TORCHIN H e ANCEL P, 2016).

Atualmente a Sociedade Brasileira de Pediatria fornece um Manual de Seguimento do Prematuro de Risco, no qual engloba um acompanhamento com equipe multidisciplinar e avaliações periódicas quanto ao desenvolvimento neuropsicomotor (DNPM), avaliação oftalmológica, avaliação com otorrinolaringologista, realização de ultrassonografias transfontanelares, avaliação nutricional, laboratorial, e quando necessárias avaliações cardiológicas (SILVEIRA RDC, 2012).

Assim, são sugeridos como imprescindíveis esses programas estruturados e especializados de seguimento dos recém-nascidos de risco (especialmente o prematuro), de modo a garantir a continuidade da assistência, promover a saúde, prevenir e identificar precocemente complicações e doenças e reduzir morbimortalidade nesses pacientes (FARIA C, et al., 2014; WHO, 2014).

Nesse sentido, os dados no Brasil ainda são escassos sobre o acompanhamento ambulatorial desses pacientes prematuros que sobrevivem ao período neonatal. Diante deste quadro, torna-se relevante a realização de estudos que identifiquem o perfil da população atendida nos Programas de Seguimento de Prematuros, para que se qualifique a assistência prestada, minimizando os efeitos que a prematuridade causa ao desenvolvimento da criança. Este estudo tem como objetivo analisar o perfil de recém-nascidos prematuros que foram atendidos em um seguimento ambulatorial em uma cidade do sul do Brasil e verificar a prevalência comorbidades. 


\section{MÉTODOS}

Trata-se de um estudo observacional, retrospectivo, através de análise de prontuários de recém-nascidos pré-termos em acompanhamento no Ambulatório de Seguimento de Prematuros do Departamento de Pediatria de uma universidade de uma cidade do sul do Brasil.

Foram consideradas elegíveis para o estudo todos os pré-termos com idade gestacional inferior a 35 semanas, que internaram em um Hospital Escola e, após alta, tiveram acompanhamento regular até completar doze meses de idade corrigida, no período de janeiro de 2010 a dezembro de 2017.

Foram excluídas as crianças com mais de 35 semanas. A coleta de dados foi realizada por acadêmicos de medicina da Faculdade de Medicina através dos prontuários médicos e de um questionário elaborado pela autora, que engloba dados de identificação, características dos prematuros, protocolo de atendimento do prematuro de risco do Ministério da Saúde e curvas de crescimento.

Foram considerados para análise os dados perinatais: sexo, idade materna, peso de nascimento, idade gestacional, apgar, tipo de parto, realização de triagem neonatal e presença de comorbidades. Os dados antropométricos obtidos no seguimento ambulatorial foram peso, perímetro cefálico e comprimento no primeiro e segundo trimestre, plotados nas curvas do intergrowth em escore Z. A idade gestacional foi determinada pela melhor estimativa obstétrica com ultrassonografia precoce e/ou data da última menstruação ou pelo exame físico do recém-nascido imediatamente pós-nascimento (CLOHERTY JP, et al., 2015). As avaliações oftalmológica e cardiológica foram realizadas respectivamente por oftalmologista, neurologista e cardiologista pediátrico.

A avaliação neurológica foi realizada por neurologista pediátrico, com no mínimo idade cronológica de seis meses. O diagnóstico de anemia e disfunção renal foram identificados por exames laboratoriais: hemograma e creatinina, respectivamente. Além disso, foi usado o exame de imagem de ultrassom transfontanelar para verificar possíveis alterações morfológicas cerebrais. Todas essas avaliações seguiram o Manual de Seguimento do Prematuro de Risco, da Sociedade Brasileira de Pediatria (SILVEIRA RDC, 2012).

A entrada dos dados foi realizada em planilha eletrônica no Excel. Inicialmente, os questionários foram codificados pelos próprios alunos e posteriormente digitados. Após a digitação ocorreu a limpeza dos dados pelos autores para tornar o banco adequado para análise. As características da amostra e prevalência de comorbidades foram descritas em frequências absoluta e relativa.

Foram inseridos no software INTERGROWTH-21st - Applications and Calculators, as médias da semana e das medidas antropométricas do primeiro e segundo trimestre para a projeção dos pontos nos gráficos (Figura 1). Para comparar as características da amostra em relação a realização da triagem neonatal e presença de morbidades, foi utilizado o teste qui-quadrado.

Para esta análise, foi utilizado o software Statistical Package for the Social Sciences (SPSS) 22.0. As associações que apresentaram um valor $\mathrm{p}<0.05$ foram consideradas estatisticamente significativas. $O$ estudo foi aprovado pelo Comitê de Ética em Pesquisa da Universidade Federal de Pelotas (número do parecer: 2.637.497), atendendo às recomendações da Resolução 196/96 do Conselho Nacional de Saúde.

\section{RESULTADOS}

Um total de 9419 prontuários foram revisados no período janeiro de 2010 a dezembro de 2017, entretanto apenas 397 preencheram os critérios de inclusão. A maioria dos recém-nascidos foram meninos $(53,1 \%)$, quase $75,0 \%$ das mães tinham entre 19-39 anos e praticamente três quartos das mães tiveram parto cesáreo (73,7\%). A maioria dos recém-nascidos nasceram com 32 semanas ou mais $(69,3 \%)$, com baixo peso $(54,4 \%)$ e obtiveram escore de apgar maior que 7 no quinto minuto $(97,2 \%)$. Na primeira consulta, quase $80 \%$ dos recém-nascidos não estavam em aleitamento materno exclusivo. Em relação a realização da triagem neonatal, mais de $85.0 \%$ dos bebês realizaram teste do pezinho e reflexo vermelho, entretanto, apenas $67,8 \%$ realizaram teste da orelhinha (Tabela $\mathbf{1}$ ). 
Tabela 1 - Características da amostra de acordo com a realização da triagem neonatal ( $n=397)$.

\begin{tabular}{|c|c|c|c|c|c|c|c|}
\hline \multirow[b]{2}{*}{ Variáveis } & \multirow{2}{*}{$\begin{array}{c}\text { Características } \\
\text { da amostra }\end{array}$} & \multicolumn{6}{|c|}{ Realização da triagem neonatal } \\
\hline & & $\begin{array}{l}\text { Teste do } \\
\text { pezinho }\end{array}$ & p-valor & $\begin{array}{c}\text { Reflexo } \\
\text { vermelho }\end{array}$ & p-valor & $\begin{array}{l}\text { Teste da } \\
\text { orelhinha }\end{array}$ & p-valor \\
\hline Sexo & & & 0,358 & & 0,428 & $133(91,7)$ & 0,262 \\
\hline Feminino & $186(46.9)$ & $158(95,8)$ & & $154(96,9)$ & & $136(87,2)$ & \\
\hline Masculino & $211(53.1)$ & $174(93,00$ & & $173(94,5)$ & & & \\
\hline Idade materna & & & 0,088 & & 0,060 & & 0,524 \\
\hline Até 18 anos & $50(12,6)$ & $34(87,2)$ & & $38(90,5)$ & & $25(83,3)$ & \\
\hline $19-39$ anos & $297(74,8)$ & $262(95,6)$ & & $259(97,0)$ & & $215(90,0)$ & \\
\hline 40 anos ou mais & $50(12,6)$ & $36(92,3)$ & & $30(90,9)$ & & $29(90,6)$ & \\
\hline Tipo de parto & & & 0,378 & & 0,497 & & 0,199 \\
\hline Normal & $98(26.3)$ & $80(93,0)$ & & $84(95,5)$ & & $66(85,7)$ & \\
\hline Cesário & $275(73.7)$ & $237(96,0)$ & & $230(97,0)$ & & $191(91,0)$ & \\
\hline Alimentação & & & 0,050 & & 0,756 & & 0,124 \\
\hline AME & $88(22,2)$ & $68(89,5)$ & & $75(94,9)$ & & $59(84,3)$ & \\
\hline Sem AME & $309(77,8)$ & $264(95,7)$ & & $252(95,8)$ & & $210(90,9)$ & \\
\hline Idade gestacional & & & 0,491 & & 0,692 & & 0,272 \\
\hline Prematuro extremo & $20(5,0)$ & $16(100,0)$ & & $15(93,8)$ & & $13(92,9)$ & \\
\hline Muito prematuro & $102(25,7)$ & $88(93,6)$ & & $82(94,3)$ & & $75(93,8)$ & \\
\hline Prematuro & $275(69,3)$ & $228(94,2)$ & & $230(96,2)$ & & $181(87,4)$ & \\
\hline Peso ao nascer & & & 0,910 & & 0,941 & & 0,363 \\
\hline$<1000 \mathrm{~g}$ & $52(13,1)$ & $43(93,5)$ & & $39(95,1)$ & & $34(91,9)$ & \\
\hline 1000 a $1499 g$ & $88(22,2)$ & $72(96,0)$ & & $71(94,7)$ & & $62(92,5)$ & \\
\hline 1500 a $2499 g$ & $216(54,4)$ & $183(93,8)$ & & $183(95,8)$ & & $147(89,1)$ & \\
\hline Acima de $2500 \mathrm{~g}$ & $41(10,3)$ & $34(94,4)$ & & $34(97,1)$ & & $26(81,3)$ & \\
\hline Apgar 5 minutos & & & 0,892 & & 0,931 & & 0,609 \\
\hline Até 7 & $24(6,0)$ & $19(95,0)$ & & $20(95,2)$ & & $14(93,3)$ & \\
\hline$>7$ & $373(94,0)$ & $313(94,3)$ & & $307(95,6)$ & & $255(89,2)$ & \\
\hline Apgar 5 minutos & & & 0,447 & & 0,365 & & 0,549 \\
\hline Até 5 & $11(2,8)$ & $9(90,0)$ & & $9(90,0)$ & & $6(85,7)$ & \\
\hline$>5$ & $386(97,2)$ & $323(94,4)$ & & $318(95,8)$ & & $263(89,5)$ & \\
\hline Total & $397(100)$ & $352(88,7)$ & & $342(86,1)$ & & $269(67,8)$ & - \\
\hline
\end{tabular}

Legenda: AME - Aleitamento Materno Exclusivo.

Fonte: Fontana F, et al., 2020; dados extraídos de uma universidade de uma cidade do sul do Brasil.

A média de semanas de nascimento, na primeira e segunda consulta, tanto para os meninos como para as meninas, foi respectivamente de 32,43 e 59 semanas. A média de peso ao nascer, $1^{\circ}$ e $2^{\circ}$ trimestre dos meninos foi $1935 \mathrm{~g}$, 3945g e $7110 \mathrm{~g}$ (Figura 1A) e nas meninas foi de $1665 \mathrm{~g}, 3550 \mathrm{~g}$ e $6365 \mathrm{~g}$ (Figura 1D). A média de cumprimento no $1^{\circ}$ e $2^{\circ}$ trimestre dos meninos foi $52 \mathrm{~cm}$ e $64,5 \mathrm{~cm}$ (Figura 1B) e nas meninas $51 \mathrm{~cm}$ e $63 \mathrm{~cm}$ (Figura 1E). A média do perímetro cefálico no $1^{\circ}$ e $2^{\circ}$ trimestre dos meninos foi de $37 \mathrm{~cm}$ e $42,5 \mathrm{~cm}$ (Figura 1C) e nas meninas foi de $36 \mathrm{~cm}$ e $41,5 \mathrm{~cm}$ (Figura 1F). 


\section{Revista Eletrônica Acervo Saúde / Electronic Journal Collection Health | ISSN 2178-2091}

Figura 1 - Médias antropométricas dos prematuros nas curvas do intergrouwth ( $n=397)$.

A

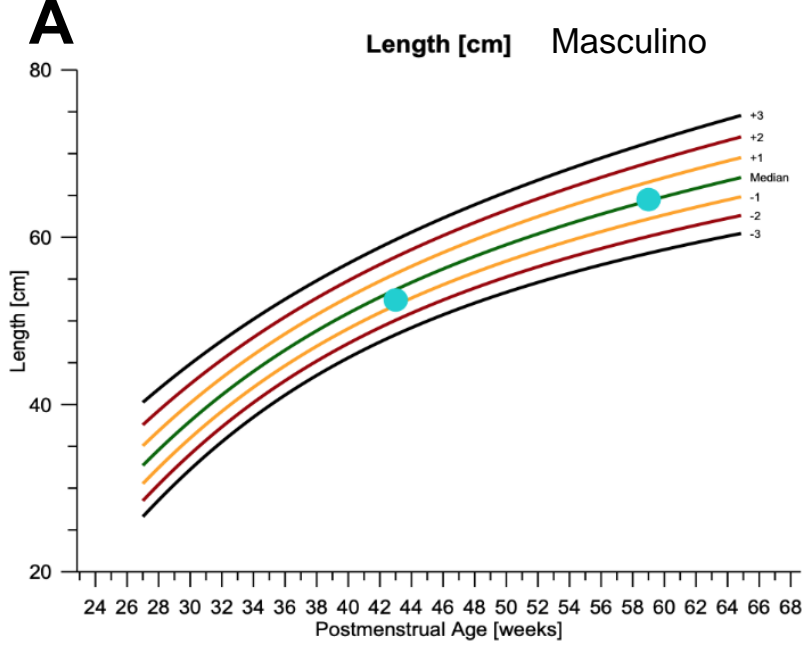

B

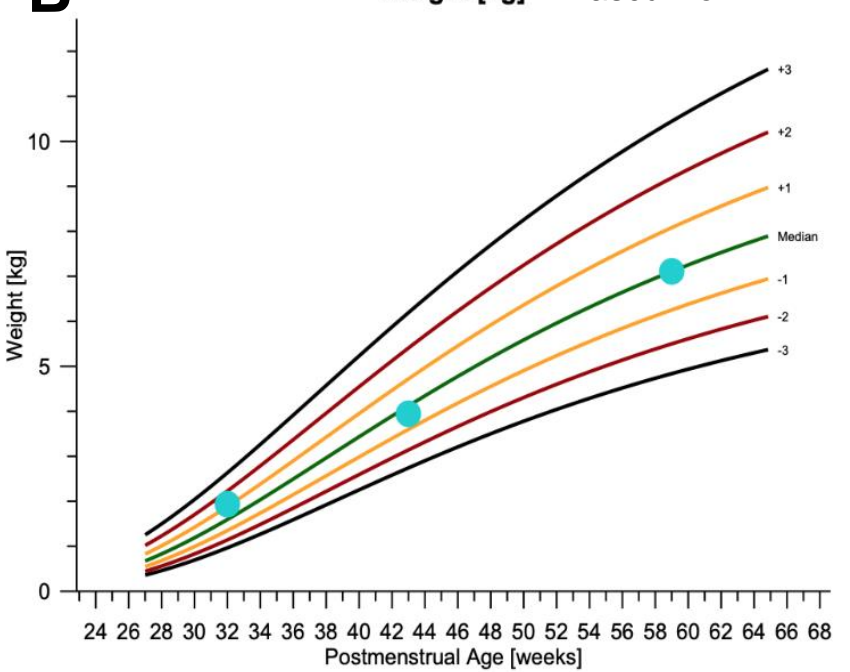

C Head circumference [cm] Masculino
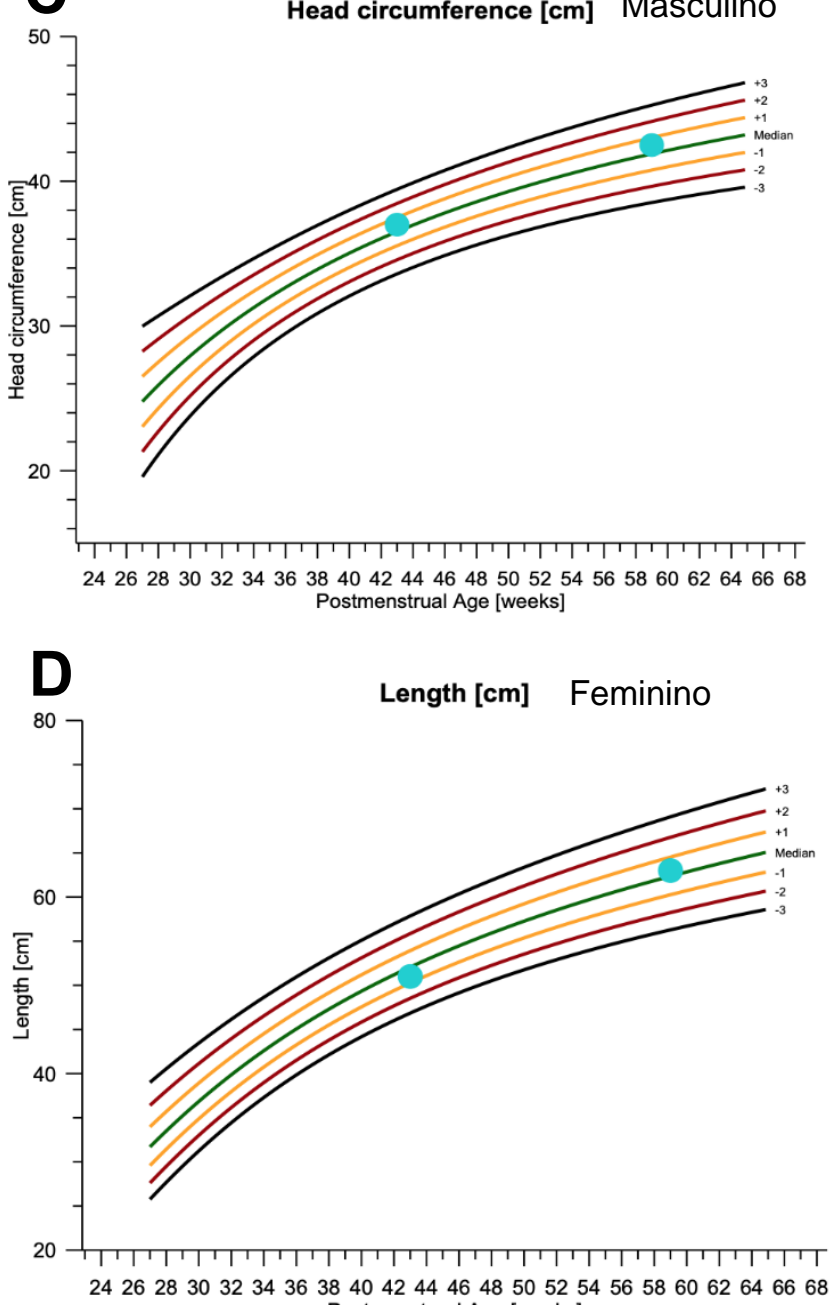

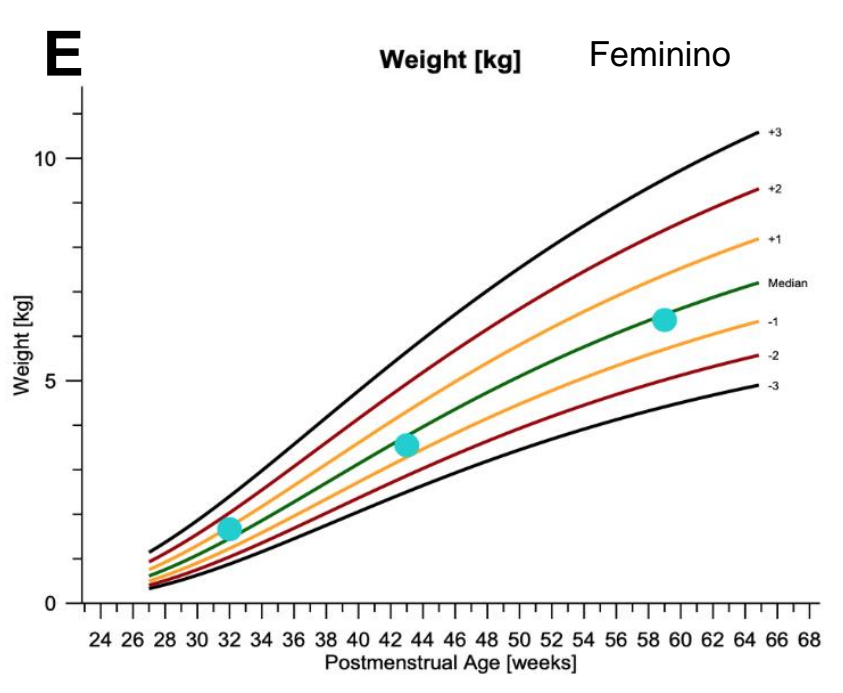

$\mathbf{F}$

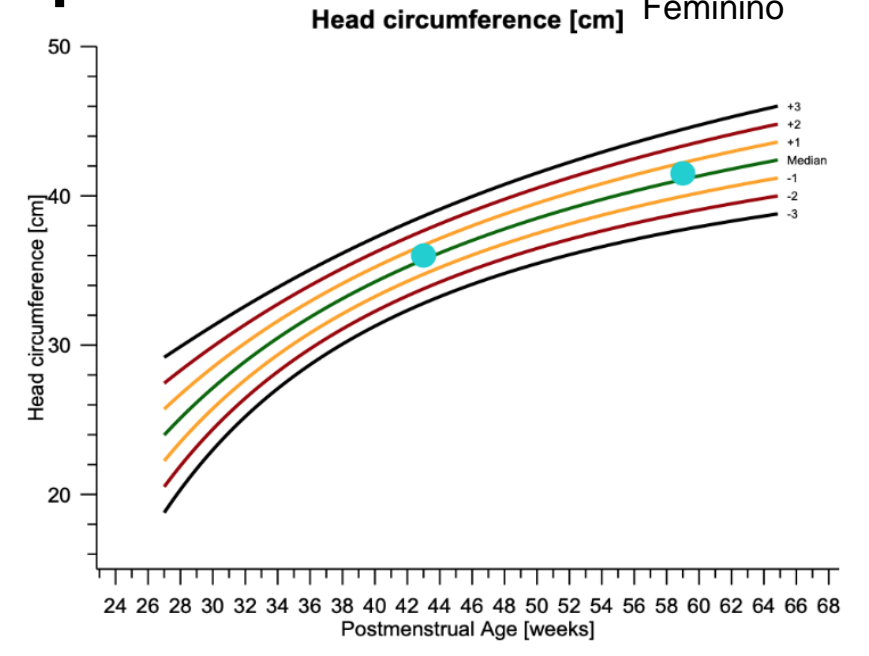

Fonte: Fontana F, et al., 2020; dados extraídos de uma universidade de uma cidade do sul do Brasil. 
As prevalências de alterações no acompanhamento dos prematuros estão descritas na Figura 2. As alterações mais prevalentes foram: Alterações cardiológicas $(51,1 \%)$, alterações neuropsicomotoras $(35,1 \%)$ e anemia $(30,5 \%)$. Na triagem neonatal, o teste da orelhinha apresentou maior alteração $(10,3 \%)$, seguidos do teste do pezinho $(3,4 \%)$ e do reflexo vermelho $(0,3 \%)$.

Figura 2 - Prevalências de alterações no acompanhamento dos prematuros ( $n=397)$.

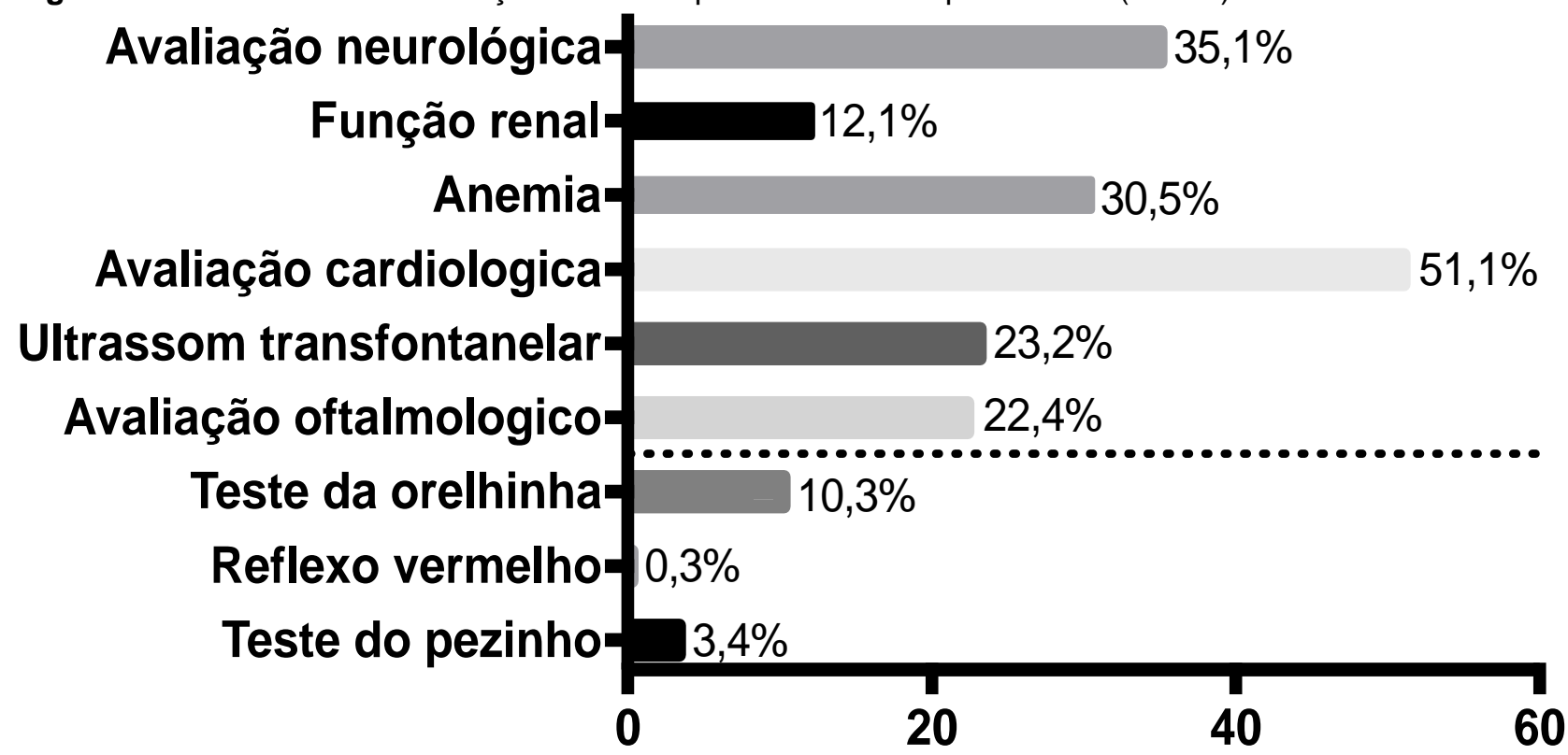

Fonte: Fontana F, et al., 2020; dados extraídos de universidade de uma cidade do sul do Brasil.

As características da amostra de acordo com a prevalência de alterações nos prematuros estão descritas na Tabela 2. Prematuridade extrema $(p<0.001)$, baixo peso ao nascer $(p<0,001)$ e apgar abaixo de 7 no quinto minuto $(p=0,041)$ foram associados a presença de alterações oftalmológicas.

As alterações neurológicas foram associadas a prematuridade extrema $(p=0,048)$ e apgar menor que 5 no quinto minuto $(p=0,048)$. O ultrassom transfontanelar foi associado ao apgar abaixo de 7 no quinto minuto $(p=0,008)$. Por fim, as disfunções renais foram significativamente maiores nas meninas que nos meninos $(p=0,013)($ Tabela 2). 


\section{Revista Eletrônica Acervo Saúde / Electronic Journal Collection Health | ISSN 2178-2091}

Tabela 2 - Características da amostra de acordo com prevalência de alterações nos prematuros $(\mathrm{n}=397)$.

\begin{tabular}{|c|c|c|c|c|c|c|c|c|c|c|c|c|}
\hline Variáveis & $\begin{array}{c}\text { Avaliação } \\
\text { oftalmológica }\end{array}$ & p-valor & $\begin{array}{l}\text { Avaliação } \\
\text { neurológica }\end{array}$ & p-valor & $\begin{array}{l}\text { Ultrassom } \\
\text { transfontanelar }\end{array}$ & p-valor & $\begin{array}{l}\text { Avaliação } \\
\text { cardiológica }\end{array}$ & p-valor & Anemia & p-valor & $\begin{array}{l}\text { Função } \\
\text { renal }\end{array}$ & p-valor \\
\hline Sexo & & 0.140 & & 0.832 & & 0.950 & & 0.702 & & 0,893 & & 0,013 \\
\hline Feminino & $22(27,5)$ & & $16(36,4)$ & & $17(17.9)$ & & $51(44,3)$ & & $24(30,0)$ & & $11(20,8)$ & \\
\hline Masculino & $15(17,6)$ & & $17(34,0)$ & & $29(28,2)$ & & $57(41,3)$ & & $34(30,9)$ & & $4(5,6)$ & \\
\hline $\begin{array}{l}\text { Idade } \\
\text { Materna }\end{array}$ & & 0,438 & & 0,961 & & 0,156 & & 0,559 & & 0,501 & & 0,994 \\
\hline Até 18 anos & $5(33,3)$ & & $2(33,3)$ & & $8(40,0)$ & & $13(52,0)$ & & $8(42,1)$ & & $1(11,1)$ & \\
\hline 19-39 anos & $26(20,3)$ & & $26(34,7)$ & & $32(20,8)$ & & $81(41,1)$ & & $44(29,5)$ & & $12(12,2)$ & \\
\hline $\begin{array}{l}40 \text { anos ou } \\
\text { mais }\end{array}$ & $6(27,3)$ & & $5(38,5)$ & & $6(25,0)$ & & $14(45,2)$ & & $6(27,3)$ & & $2(11,8)$ & \\
\hline Tipo de parto & & 0.387 & & 0.484 & & 0.326 & & & & 0,856 & & 0,550 \\
\hline Normal & $11(26,8)$ & & $8(26,6)$ & & $14(29,2)$ & & $25(38,3)$ & & $14(28,6)$ & & $5(15,2)$ & \\
\hline Cesário & $24(29,2)$ & & $24(36,9)$ & & $31(21,5)$ & & $79(45,1)$ & & $42(31,3)$ & & $10(11,4)$ & \\
\hline Alimentação & & 0.326 & & 1.124 & & 0.678 & & 0.218 & & 0,089 & & 0,741 \\
\hline AME & $4(13,8)$ & & $4(20,0)$ & & $8(19,5)$ & & $19(34,5)$ & & $18(41,9)$ & & $3(10,3)$ & \\
\hline Sem AME & $33(24,3)$ & & $29(39,2)$ & & $38(24,2)$ & & $89(44,9)$ & & $40(27,2)$ & & $12(12,6)$ & \\
\hline
\end{tabular}


Revista Eletrônica Acervo Saúde / Electronic Journal Collection Health | ISSN 2178-2091

\begin{tabular}{|c|c|c|c|c|c|c|c|c|c|c|c|c|}
\hline Variáveis & $\begin{array}{c}\text { Avaliação } \\
\text { oftalmológica }\end{array}$ & p-valor & $\begin{array}{c}\text { Avaliação } \\
\text { neurológica }\end{array}$ & p-valor & $\begin{array}{l}\text { Ultrassom } \\
\text { transfontanelar }\end{array}$ & p-valor & $\begin{array}{l}\text { Avaliação } \\
\text { cardiológica }\end{array}$ & p-valor & Anemia & p-valor & $\begin{array}{l}\text { Função } \\
\text { renal }\end{array}$ & p-valor \\
\hline $\begin{array}{c}\text { Idade } \\
\text { Gestacional }\end{array}$ & & $<0,001$ & & 0,048 & & 0,825 & & 0,094 & & 0,838 & & 0,107 \\
\hline $\begin{array}{l}\text { Prematuro } \\
\text { extremo }\end{array}$ & $9(75,0)$ & & $7(70,0)$ & & $4(26,7)$ & & $10(62,5)$ & & $5(41,7)$ & & $1(14,3)$ & \\
\hline $\begin{array}{l}\text { Muito } \\
\text { prematuro }\end{array}$ & $25(37,0)$ & & $8(28,6)$ & & $17(25,0)$ & & $36(48,0)$ & & $18(31,0)$ & & $9(20,0)$ & \\
\hline Prematuro & $3(3,4)$ & & $18(32,1)$ & & $25(21,7)$ & & $62(38,3)$ & & $35(29,3)$ & & $5(6,9)$ & \\
\hline $\begin{array}{c}\text { Peso de } \\
\text { nascimento } \\
(\mathrm{g})\end{array}$ & & $<0,001$ & & 0,496 & & 0,443 & & 0,076 & & 0,885 & & 0,868 \\
\hline$<1000 \mathrm{~g}$ & $23(67,6)$ & & $8(44,4)$ & & $10(27,0)$ & & $17(48,6)$ & & $9(34,6)$ & & $3(17,6)$ & \\
\hline 1000 a $1499 \mathrm{~g}$ & $13(25,0)$ & & $11(40,7)$ & & $15(26,3)$ & & $34(54,8)$ & & $15(29,4)$ & & $5(12,8)$ & \\
\hline 1500 a $2499 g$ & $1(1,5)$ & & $12(30,8)$ & & $20(22,2)$ & & $46(35,7)$ & & $30(31,3)$ & & $6(10,3)$ & \\
\hline $\begin{array}{c}\text { Acima de } \\
2500 \mathrm{~g}\end{array}$ & $0(0,0)$ & & $2(20,0)$ & & $1(7,1)$ & & $11(40,7)$ & & $4(23,5)$ & & $1(10,0)$ & \\
\hline $\begin{array}{l}\text { Apgar } 5 \\
\text { minutos }\end{array}$ & & 0.041 & & 0.124 & & 0.008 & & 0.301 & & 0,205 & & 0,925 \\
\hline Até 7 & $5(45,5)$ & & $5(62,5)$ & & $8(53,8)$ & & $9(56,3)$ & & $6(46,2)$ & & $1(11,1)$ & \\
\hline Maior que 7 & $32(20,8)$ & & $28(32,6)$ & & $38(20,8)$ & & $99(41,8)$ & & $52(29,4)$ & & $14(12,2)$ & \\
\hline $\begin{array}{c}\text { Apgar } 5 \\
\text { minutos } \\
\text { score }\end{array}$ & & 0.127 & & 0.048 & & 0.086 & & 1.000 & $2(40,0)$ & 0,641 & & 0,515 \\
\hline Até 5 & $3(50,0)$ & & $4(80,0)$ & & $4(40,0)$ & & $3(37,5)$ & & $56(30,3)$ & & $0(0,0)$ & \\
\hline Maior que 5 & $3421,4)$ & & $29(32,6)$ & & $42(22,1)$ & & $105(42,9)$ & & & & $15(12,4)$ & \\
\hline
\end{tabular}

Legenda: AME, Aleitamento Materno Exclusivo.

Fonte: Fontana F, et al., 2020; dados extraídos de universidade de uma cidade do sul do Brasil. 


\section{DISCUSSÃO}

Os resultados do presente estudo investigando o crescimento de crianças prematuras no primeiro ano de vida revelaram que, de forma geral, as crianças prematuras de ambos os sexos demonstraram crescimento compatível com as curvas do intergrowth nos seis primeiros meses de vida. As curvas de referência, como a do intergrowth-21, representam um padrão de crescimento supostamente normal de uma população, que podem ser utilizados para avaliar e monitorar o bem estar materno e fetal, assim como a saúde e o bem-estar infantil, tanto a nível individual como populacional (BARRETO CM, et al., 2020).

Os parâmetros normais dentro dessa curva significam que, de maneira geral, os pré-termos avaliados neste estudo se desenvolvem em boas condições para o crescimento, o que poderá estar relacionado com crescimento normal na infância e adolescência (BARRETO CM, et al., 2020). O Aleitamento Materno Exclusivo (AME) é de extrema importante para o crescimento normal e nos prematuros é considerada ainda mais relevante. No nosso estudo, foi verificado na $1^{\text {a }}$ consulta após a alta hospitalar uma prevalência $22,0 \%$ de AME.

Essa frequência pode ser considerada baixa se for comparada com outros estudos (BRIERE CE, et al., 2014; MENEZES MADS, et al., 2014). Autores salientam que os recém nascidos pré-termo que estão prontos para sugar devem ser estimulados aleitamento materno, pois sabe-se que a incidência e a gravidade de comorbidades estão significativamente diminuídas nos prematuros que foram alimentados exclusivamente com leite materno ou receberam pelo menos $80 \%$ da sua ingestão láctea na forma de leite humano (NASCIMENTO MBRD e ISSLER H, 2004).

Segundo a literatura, a orientação sobre amamentação revela-se uma importante ação em prol dessa prática, e deve ser proporcionada às mães de prematuros durante a hospitalização, dando-se continuidade a essa atividade após a alta (COUTO FF e PRAÇA NDS, 2009; SANTORO JÚNIOR W e MARTINEZ FE, 2007). Salienta-se que, embora seja conhecido a importância do incentivo ao aleitamento materno durante a internação hospitalar, não obtivemos dados sobre tal fato em nossa amostra.

É bem reconhecido a importância da triagem neonatal como ação preventiva que permite fazer o diagnóstico de diversas doenças congênitas ou infecciosas e assintomáticas no período neonatal (BRASIL, 2016). A triagem neonatal se baseia na realização de testes laboratoriais nos primeiros dias de vida do recémnascido, que se realizados no momento e da forma adequados permitem que o início do tratamento ocorra dentro de uma janela de tempo em que é possível evitar sequelas no desenvolvimento da criança (SILVEIRA RDC, 2012).

No presente estudo, mais de $85.0 \%$ dos pré-termos realizaram o teste do pezinho e reflexo vermelho e $67 \%$ realizaram o teste da orelhinha. Estudo prévio mostra que a prevalência de realização do teste do pezinho no Brasil em qualquer momento de vida foi de $96,5 \%$; do teste da orelhinha de $65,8 \%$ e do teste do olhinho de 60,4\% (MALLMANN MB, et al., 2020).

O resultado do teste do olhinho superior ao estudo de Mallmann MB, et al. (2020), pode ser justificado pela presença do pediatra no atendimento da sala de parto de todos os recém-nascidos do hospital e o mesmo ser capacitado para realização do teste do olhinho. Neste estudo, alterações cardiológicas, neurológicas e presença de anemia foram as morbidades mais prevalentes. Alteração na função renal e oftalmológicas também foram identificadas, com menor prevalência, mas de igual modo importantes para o crescimento e desenvolvimento adequado dos prematuros.

Em mais da metade dos prematuros avaliados foi identificado alterações cardiológicas, resultado concordante com o descrito na literatura (AMORIM LF, et al., 2008; LOPES SAVDA, et al., 2018). Este fato é explicado porque a circulação fetal sofre uma transição dramática no nascimento, e no caso dos prematuros, esta transição ocorre em um estágio crítico do desenvolvimento de órgãos (DE WAAL K, et al., 2016). Carr $\mathrm{H}$, et al. (2017) em um grande estudo de coorte baseado em registro que incluiu 2,67 milhões de indivíduos nascidos na Suécia de 1987 a 2012 mostram que os indivíduos nascidos prematuramente têm maior risco de insuficiência cardíaca incidente desde a infância até a idade adulta jovem, com um risco quatro vezes maior naqueles nascidos com 28-31 semanas de gestação (muito prematuro) e risco 17 vezes maior em crianças nascidas com <28 semanas de gestação (prematuro extremo). 
Assim, os prematuros com sinais de alterações cardiovasculares devem ser submetidos a avaliação cardiológica e acompanhamento adequado se diagnosticada alguma malformação cardíaca (AMORIM LF, et al., 2008). Problemas no coração são a terceira maior causa de morte em recém-nascidos. Por isso, quanto mais cedo for diagnosticado, melhores são as chances do tratamento (SILVEIRA RDC, 2012).

Além disso, os estudos atuais enfatizam que a prematuridade é um dos principais fatores de risco biológico para o atraso no desenvolvimento neuropsicomotor, sendo que o ultrassom transfontanelar é um grande aliado para diagnóstico de complicações da prematuridade, como o desenvolvimento de hemorragia intracraniana, mais prevalente quanto menor a idade gestacional de nascimento (WOYTHALER MA, et al., 2011; RESEGUE R, et al., 2008; FARAGE L e ASSIS MC, 2005; KRELING K, et al., 2006).

Foi observado que $35,1 \%$ das crianças deste estudo apresentaram anormalidade no desenvolvimento, com maior frequência entre os bebês prematuros extremo. Resultado semelhante também foi encontrado no estudo de Halpern R, et al. (2002), onde identificaram teste indicativo de atraso no desenvolvimento em 34\% da amostra e um risco três vezes maior entre as crianças que nasceram com peso inferior a 2.000 gramas.

Estudos anteriores destacam que os sistemas imaturos de órgãos comuns no parto do prematuro também podem levar a dificuldades na adaptação a diferentes estímulos ambientais e aumentar as chances de o bebê apresentar distúrbios neurológicos durante vários estágios de desenvolvimento (HALPERN R, et al., 2002; RESEGUE R, et al., 2008). Além disso, o achado de Apgar baixo no quinto minuto entre os pré-termos que apresentaram atraso no desenvolvimento reforça a importância do diagnóstico e do acompanhamento prénatal adequados. Entretanto, devido a essa complexa interação entre fatores biológicos e ambientais, prever o curso do desenvolvimento nessa população não é uma tarefa fácil.

A prevalência de anemia foi relativamente baixa neste estudo quando comparada um uma revisão sistemática incluindo lactantes a termo e pré-termo no Brasil, cuja prevalência foi de 53 , possivelmente porque as crianças são acompanhadas no seguimento ambulatorial vinculado a alta hospitalar \% (JORDÃO RE, et al., 2009). Além disso, é rotina a suplementação regular de ferro e vitaminas desde a alta hospitalar e durante todo o primeiro ano de vida.

Ainda assim, essa prevalência é superior aos países desenvolvidos, com relatos de prevalências que variam entre $3 \%$ a 5\% em lactantes de 2000-2500 grama (BERGLUND S, et al., 2010). O fato de avaliarmos uma população de pré-termos explica pelo menos em parte este resultado, pois as reservas de ferro estão reduzidas em decorrência do baixo peso ao nascer, dificultando a produção de hemoglobina (CLOHERTY JP, 2015). Além desses órgãos-chave associados a complicações do parto prematuro, distúrbios ao nível oftalmológico também foram encontrados. A incidência de retinopatia da prematuridade é uma grande preocupação no parto prematuro.

A Retinopatia da Prematuridade é uma doença de etiologia multifatorial, vasoproliferativa secundária à vascularização inadequada da retina imatura dos recém-nascidos pré-termos (RNPT) (TOMÉ VAV, et al., 2011). Estima-se que haja 1,5 milhões de crianças cegas no mundo, sendo que a ROP continua sendo importante causa de cegueira infantil principalmente na América Latina. Em países de renda média, esse número é muito variável, dependendo das taxas de nascimento e de sobrevivência de recém-nascidos prétermo e da existência de programas de triagem para detecção (ZIN A e GLEN AG, 2013; GILBERT C, et al., 1997).

Em nosso estudo, o peso ao nascer e menor tempo de gestação se mostraram associados a alterações oftalmológicas, o que é consistente com estudos prévios, que identificam a prematuridade e o baixo peso ao nascer como os principais fatores de risco para o desenvolvimento de retinopatia na prematuridade (STUTCHFIELD C, et al., 2017; TOMÉ VAV, et al., 2011).

Também foi observado que o grupo com alteração oftalmológica apresentou escores mais baixas no Apgar-5 comparado ao grupo sem alterações oftalmológicas. Assim como neste estudo, escore Apgar-5 menor que 7 apresentou-se como um fator de risco para a retinopatia na prematuridade em outros estudos (BONOTTO LB, et al., 2007; GRAZIANO RM e LEONE CR, 2005). 
Por fim, curiosamente encontramos uma associação significativa entre a disfunção renal e sexo feminino. É conhecido que há número reduzido de néfrons em recém-nascidos prematuramente, no qual pode predispor estes pacientes ao desenvolvimento de insuficiência renal, pois o número de néfrons e a taxa de filtração glomerular de cada néfron estão reduzidos nos bebês prematuros (LIBÓRIO AB, et al., 2014; BOLAT F, et al., 2013). Entretanto, o resultado de alterações renais prevalente nas meninas é divergente com estudo prévio, o qual revelou que o sexo masculino foi associado a um risco 1,3 vezes maior de doença renal em comparação ao sexo feminino (CRUMP C, 2019). Temos como hipótese o $\mathrm{n}$ amostral muito baixo de disfunções renais, o que pode ter confundido os resultados. Mais estudos com número maior de pacientes são necessários para verificação desse resultado.

Os resultados devem ser interpretados considerando algumas limitações. Apesar de o crescimento ser também influenciado por fatores socioeconômicos e nutricionais maternos, no presente estudo não se pôde verificar essa influência. Não obtivemos dados sobre o período de internação hospitalar, uma vez que essas informações não constavam nos prontuários revisados. Outro problema encontrado foi o preenchimento incompleto ou ilegível dos prontuários médicos. O caráter transversal do estudo não permitiu inferências causais. Entretanto, aspectos positivos devem ser ressaltados, como a aplicação das medidas antropométricas dos recém-nascidos na curva internacional de crescimento Intergrowth-21st e os poucos estudos nacionais que são publicados explorando as características, realização da triagem neonatal e comorbidades dos recém-nascidos pré-termos, revelando uma escassez de dados sobre o acompanhamento ambulatorial de pacientes prematuros que sobrevivem ao período neonatal.

\section{CONCLUSÃO}

Em conclusão, os resultados emergentes do presente estudo sugerem que os prematuros acompanhados no ambulatório de pediatria têm um crescimento adequado e compatível com as curvas do Intergrowth-21st. As prevalências de comorbidades estão convergentes com a população de prematuros acompanhados por outros estudos. Prematuridade extrema, baixo peso ao nascer e apgar abaixo de 7 no quinto minuto foram associados a presença de alterações oftalmológicas, bem como ser prematuro extremo foi associado a alterações neurológicas. Apgar menor que 7 no quinto minuto foi associado apenas com alteração de imagem no ultrassom transfontanelar. Sendo assim, é imprescindível o acompanhamento a médio e longo prazo desses pacientes, especialmente nos casos de prematuridade extrema, baixo peso ao nascer e apgar baixo para diagnóstico e intervenção precoce das morbidades. Neste estudo foram fornecidos elementos para conhecer o perfil e a demanda dos cuidados requeridos por essas crianças e proporcionar uma adequada qualidade de vida a esses prematuros.

\section{REFERÊNCIAS}

1. AMORIM LF, et al. Apresentação das cardiopatias congênitas diagnosticadas ao nascimento: análise de 29.770 recém-nascidos. Jornal de Pediatria. 2008; 84(1): 83-90.

2. BARRETO CM, et al. Incidence of small for gestational age neonates, according to the fenton and intergrowth-21st curves in a level ii maternity. Revista Paulista de Pediatria. 2021; 39.

3. BERGLUND S, et al. Iron supplements reduce the risk of iron deficiency anemia in marginally low birth weight infants. Pediatrics. 2010; 126(4): e874-e883.

4. BOLAT F, at al. Acute kidney injury in a single neonatal intensive care unit in Turkey. World Journal of Pediatrics. 2013; 9(4): 323-329.

5. BONOTTO LB, et al.. Prevalência de retinopatia da prematuridade em prematuros atendidos no período de $1992-$ 1999 em Joinville (SC): avaliação de riscos associados-" screening". Arquivos brasileiros de oftalmologia. 2007; 70(1): 55-61.

6. BRASIL LN. Ministério da Saúde. Secretaria de Atenção à Saúde. Departamento de Atenção Especializada e Temática. Manual de orientações para promoção da doação voluntária de sangue/Ministério da Saúde, Secretaria de Atenção a Saúde Departamento de Atenção Especializada e Temática-. 2016; 1:17.

7. BRIERE CE, et al. An integrative review of factors that influence breastfeeding duration for premature infants after NICU hospitalization. Journal of Obstetric, Gynecologic \& Neonatal Nursing. 2014; 43(3): 272-281.

8. CARR $\mathrm{H}$, et al. Preterm birth and risk of heart failure up to early adulthood. Journal of the American College of Cardiology. 2017; 69(21): 2634-2642.

9. CLOHERTY JP, et al. Manual de neonatologia. In: Manual de neonatologia. 2005: 715-715.

10. COUTO FF, PRAÇA NDS. Preparo dos pais de recém-nascido prematuro para alta hospitalar: uma revisão bibliográfica. Escola Anna Nery. 2009;13(4):886-892. 
11. CRUMP C, et al. Preterm birth and risk of chronic kidney disease from childhood into mid- adulthood: national cohort study. bmj. 2019; 365.

12. DANI C, et al. The role of blood transfusions and iron intake on retinopathy of prematurity. Early human development. 2001; 62(1): 57-63.

13. DE WAAL $\mathrm{K}$, et al. Cardiac function after the immediate transitional period in very preterm infants using speckle tracking analysis. Pediatric Cardiology. 2016; 37(2): 295-303.

14. FARAGE L, ASSIS MC. Achados ultra-sonográficos da hemorragia intracraniana em recém-nascidos prematuros. Arquivos de Neuro-Psiquiatria. 2005; 63(3b): 814-816.

15. FARIA C, et al. Morbidity and mortality among the high-risk newborns: a bibliography review. Enferm Global. 2014; 36: 298-309.

16. GILBERT C, et al. Retinopathy of prematurity in middle-income countries. The Lancet. 1997; 350(9070): 12-14.

17. GRAZIANO RM, LEONE CR. Problemas oftalmológicos mais freqüentes e desenvolvimento visual do pré-termo extremo. Jornal de Pediatria. 2005; 81(1): S95-S100.

18. HALPERN R, et al. Fatores de risco para suspeita de atraso no desenvolvimento neuropsicomotor aos 12 meses de vida. Revista chilena de pediatría. 2002; 73(5): 529-539.

19. HEDDERICH DM, et al. Aberrant gyrification contributes to the link between gestational age and adult IQ after premature birth. Brain. 2019; 142(5): 1255-1269.

20. HEDDERICH DM, et al. Sequelae of Premature Birth in Young Adults: Incidental Findings on Routine Brain MRI. Clinical Neuroradiology. 2020: 1-9.

21. JORDÃO RE, et al. Prevalência de anemia ferropriva no Brasil: uma revisão sistemática. Revista Paulista de Pediatria. 2009; 27(1): 90-98.

22. KRELING K, et al.. Fatores perinatais associados ao desenvolvimento neuropsicomotor de recém-nascidos de muito baixo peso. Pediatria (São Paulo). 2006; 28(2): 98-108.

23. LEHTONEN L, et al. Early neonatal death: a challenge worldwide. Paper presented at: Seminars in Fetal and Neonatal Medicine. 2017.

24. LIBÓRIO AB, et al. Acute kidney injury in neonates: from urine output to new biomarkers. BioMed research international. 2014;2014.

25. LOPES SAVDA, et al. Mortalidade para Cardiopatias Congênitas e Fatores de Risco Associados em RecémNascidos. Um Estudo de Coorte. Arquivos Brasileiros de Cardiologia. 2018; 111(5): 666-673.

26. MAIA RRP, SOUZA JMP. Fatores associados ao baixo peso ao nascer em município do norte do Brasil. Journal of Human Growth and Development. 2010; 20(3): 735-744.

27. MALLMANN MB, et al. Neonatal screening tests in Brazil: prevalence rates and regional and socioeconomic inequalities. Jornal de Pediatria (Versão em Português). 2020; 96(4): 487-494.

28. MENEZES MADS, et al. Preterm newborns at Kangaroo Mother Care: a cohort follow-up from birth to six months. Revista Paulista de Pediatria. 2014; 32: 171-177.

29. MOREIRA NETO AR, et al. Etiologia da restrição de crescimento intrauterino (RCIU). Comunicação em Ciências da Saúde. 2011:21-30.

30. NASCIMENTO MBRD, ISSLER H. Aleitamento materno em prematuros: manejo clínico hospitalar. Jornal de Pediatria. 2004; 80(5): s163-s172.

31. RESEGUE R, et al. Risk factors associated with developmental abnormalities among high-risk children attended at a multidisciplinary clinic. São Paulo Medical Journal. 2008; 126(1): 4-10.

32. SAFARI S, HAMRAH MP. Epidemiology and related risk factors of preterm labor as an obstetrics emergency. Emergency. 2017; 5(1).

33. SANTORO JÚNIOR W, MARTINEZ FE. Impacto de uma intervenção pró-aleitamento nas taxas de amamentação de recém-nascidos de muito baixo peso. Jornal de Pediatria. 2007; 83(6): 541-546.

34. SILVEIRA RDC. Manual seguimento ambulatorial do prematuro de risco. Porto Alegre: Sociedade Brasileira de Pediatria. 2012; 1.

35. STUTCHFIELD C, et al. Foetal haemoglobin, blood transfusion, and retinopathy of prematurity in very preterm infants: a pilot prospective cohort study. Eye. 2017; 31(10): 1451-1455.

36. TOMÉ VAV, et al. Study of retinopathy of prematurity in a university hospital. Arquivos brasileiros de oftalmologia. $2011 ; 74(4)$ : 279-282.

37. TORCHIN H, ANCEL P. Epidemiology and risk factors of preterm birth. Journal de gynecologie, obstetrique et biologie de la reproduction. 2016; 45(10): 1213.

38. WHO. WHO recommendations on postnatal care of the mother and newborn. World Health Organization; 2014.

39. WOYTHALER MA, et al. Late preterm infants have worse 24-month neurodevelopmental outcomes than term infants. Pediatrics. 2011; 127(3): e622-e629.

40. ZIN A, GLEN AG. Retinopathy of prematurity-incidence today. Clinics in perinatology. 2013; 40 (2): $185-200$. 